\title{
Pathophysiological Mechanisms of Dominant and Recessive GLRA1 Mutations in Hyperekplexia
}

\author{
Seo-Kyung Chung, ${ }^{1}$ Jean-François Vanbellinghen, ${ }^{2}$ Jonathan G. L. Mullins, ${ }^{1}$ Angela Robinson, ${ }^{1}$ Janina Hantke, ${ }^{3}$ \\ Carrie L. Hammond, ${ }^{1}$ Daniel F. Gilbert, ${ }^{4}$ Michael Freilinger, ${ }^{5}$ Monique Ryan, ${ }^{6}$ Michael C. Kruer, ${ }^{7}$ Amira Masri, ${ }^{8}$ \\ Candan Gurses, ${ }^{9}$ Colin Ferrie, ${ }^{10}$ Kirsten Harvey, ${ }^{3}$ Rita Shiang, ${ }^{11}$ John Christodoulou, ${ }^{12,13}$ Frederick Andermann, ${ }^{14}$ \\ Eva Andermann, ${ }^{14}$ Rhys H. Thomas, ${ }^{1}$ Robert J. Harvey, ${ }^{3}$ Joseph W. Lynch, ${ }^{4}$ and Mark I. Rees ${ }^{1}$ \\ ${ }^{1}$ Institute of Life Science, School of Medicine, Swansea University, Swansea SA2 8PP, United Kingdom, ${ }^{2}$ Laboratoire de Génétique Moléculaire, University \\ of Liège, Liège, Centre Hospitalier Universitaire-Tour de Pathologie B23 +3, 4000 Sart Tilman, Belgium, ${ }^{3}$ Department of Pharmacology, School of \\ Pharmacy, London WC1N 1AX, United Kingdom, ${ }^{4}$ Queensland Brain Institute and School of Biomedical Sciences, University of Queensland, Brisbane, \\ Queensland 4072, Australia, ${ }^{5}$ Department of Pediatrics and Adolescent Medicine, Medical University of Vienna, 1090 Vienna, Austria, ${ }^{6}$ Children's \\ Neurosciences Centre, Murdoch Childrens Research Institute, Royal Children's Hospital, Parkville, Victoria 3052, Australia, ${ }^{7}$ Divisions of Developmental \\ Pediatrics and Pediatric Neurology, Child Development and Rehabilitation Center, Oregon Health and Science University, Portland, Oregon 97239, \\ ${ }^{8}$ Department of Pediatrics, Faculty of Medicine, University of Jordan, Amman 11942, Jordan, ${ }^{9}$ Department of Neurology, Istanbul University, Millet Cd \\ Capa 34390, Istanbul, Turkey, ${ }^{10}$ Department of Paediatric Neurology, Leeds General Infirmary, Leeds LS2 9NS, United Kingdom, ${ }^{11}$ Department of Human \\ and Molecular Genetics, Virginia Commonwealth University, Richmond, Virginia 23298-0033, ${ }^{12}$ Western Sydney Genetics Program, Children's Hospital, \\ Westmead, New South Wales 2145, Australia, ${ }^{13}$ Disciplines of Paediatrics and Child Health and Genetic Medicine, Sydney Medical School, University of \\ Sydney, Sydney, New South Wales 2145, Australia, and ${ }^{14}$ Montreal Neurological Hospital and Institute, McGill University, Montreal, Quebec H3A 2B4, \\ Canada
}

Hyperekplexia is a rare, but potentially fatal, neuromotor disorder characterized by exaggerated startle reflexes and hypertonia in response to sudden, unexpected auditory or tactile stimuli. This disorder is primarily caused by inherited mutations in the genes encoding the glycine receptor (GlyR) $\alpha 1$ subunit (GLRA1) and the presynaptic glycine transporter GlyT2 (SLC6A5). In this study, systematic DNA sequencing of GLRA1 in 88 new unrelated human hyperekplexia patients revealed 19 sequence variants in 30 index cases, of which 21 cases were inherited in recessive or compound heterozygote modes. This indicates that recessive hyperekplexia is far more prevalent than previous estimates. From the 19 GLRA1 sequence variants, we have investigated the functional effects of 11 novel and 2 recurrent mutations. The expression levels and functional properties of these hyperekplexia mutants were analyzed using a high-content imaging system and patch-clamp electrophysiology. When expressed in HEK293 cells, either as homomeric $\alpha 1$ or heteromeric $\alpha 1 \beta$ GlyRs, subcellular localization defects were the major mechanism underlying recessive mutations. However, mutants without trafficking defects typically showed alterations in the glycine sensitivity suggestive of disrupted receptor function. This study also reports the first hyperekplexia mutation associated with a GlyR leak conductance, suggesting tonic channel opening as a new mechanism in neuronal ligand-gated ion channels.

\section{Introduction}

Glycine receptors (GlyRs) are members of the pentameric Cysloop ion channel superfamily and major determinants of inhibitory neurotransmission in the retina, spinal cord, and brainstem (Lynch, 2004). Each GlyR subunit comprises a large N-terminal ligand-binding domain (LBD) and membrane-spanning region comprising four $\alpha$-helical domains (M1-M4). At least four GlyR subunits exist in the human brain $(\alpha 1, \alpha 2, \alpha 3$, and $\beta)$, assembling

\footnotetext{
Received April 7, 2010; revised May 26, 2010; accepted June 4, 2010.

This work was supported by Medical Research Council United Kingdom Grant G0601585 (M.I.R., K.H., R.J.H.), the Wales Office of Research and Development (M.I.R.), and the National Health and Medical Research Council of Australia (J.W.L.). We also thank Dr. Tim Webb for his assistance in laboratory methodology.

Correspondence should be addressed to Prof. Mark I. Rees, Institute of Life Science, Swansea University, Singleton Park, Swansea SA2 8PP, UK. E-mail: m.i.rees@swansea.ac.uk.

DOI:10.1523/JNEUROSCI.1763-10.2010

Copyright $\odot 2010$ the authors $\quad 0270-6474 / 10 / 309612-09 \$ 15.00 / 0$
}

predominantly as $\alpha 1_{2} \beta_{3}$ heteropentamers (Lynch, 2004; Grudzinska et al., 2005). Mutations in the GlyR $\alpha 1$ (GLRA1) and GlyR $\beta(G L R B)$ subunit genes are associated with startle disease/hyperekplexia with 29 distinct GLRA1 mutations in 56 index cases and families described (OMIM: 149400) (Shiang et al., 1993; Rees et al., 2002; Harvey et al., 2008). Recently, defects in a presynaptic glycine transporter gene (SLC6A5) have been identified in human hyperekplexia (Rees et al., 2006).

Hyperekplexia is a rare, but potentially fatal, neurological disorder characterized by an abnormal startle reflex and an exaggerated muscle stiffness in response to sudden, unexpected auditory or tactile stimuli (Harvey et al., 2008). The symptoms are most severe in neonates and infants in whom intense hypertonicity can lead to apnea episodes and prolonged cyanotic attacks. The hypertonia, which is predominantly in the trunk and lower limbs, often diminishes during the first year of life, but the pathological 
Table 1. Details of hyperekplexia mutations identified in this study

\begin{tabular}{|c|c|c|c|c|c|c|c|c|}
\hline Case & Sequence & $\begin{array}{l}\text { Classification of } \\
\text { mutation }\end{array}$ & Mutants & & Protein position & Gender & Mode of inheritance & References \\
\hline $1-6$ & $\Delta$ Exons 1-7 (Homo) & Deletion & del Ex1-7 & & NA & $3 \mathrm{~F}, 3 \mathrm{M}$ & $\mathrm{R}$ & $\begin{array}{l}\text { Brune et al., 1996; } \\
\quad \text { Gilbert et al., } 2004\end{array}$ \\
\hline \multirow[t]{2}{*}{7} & G574T & Missense & R65L & Mat & $\mathrm{N}$-terminal & & \multirow{2}{*}{$R$} & Novel \\
\hline & $\Delta$ Exons 4-7 & Deletion & del Ex4-7 & Pat & NA & M & & Novel \\
\hline 8 & С573T (Homo) & Missense & R65W & & N-terminal & M & $\mathrm{R}$ & Novel \\
\hline 9 & $\Delta 931-932$ CT (Homo) & Deletion & L184fs21X & & $\mathrm{N}$-terminal & M & $\mathrm{R}$ & Novel \\
\hline \multirow[t]{2}{*}{10} & $\Delta 931-932 C T$ & Deletion & L184fs21X & Pat & N-terminal & \multirow{2}{*}{ M } & R & Novel \\
\hline & G687A & Missense & E103K & Mat & $\mathrm{N}$-terminal & & $\mathrm{R}$ & Novel \\
\hline 11 & A764G & Missense & Y128C & & N-terminal & M & $D$ & Novel \\
\hline 12 & A874G & Missense & D165G & & N-terminal & $\mathrm{F}$ & $\mathrm{R}$ & Novel \\
\hline \multirow{2}{*}{13} & C971A & Nonsense & Y197X & Pat & N-terminal & \multirow{2}{*}{$M$} & \multirow{2}{*}{$\mathrm{R}$} & Novel \\
\hline & C986A & Nonsense & Y202X & Mat & $\mathrm{N}$-terminal & & & Rees et al., 2001 \\
\hline 14 & C971A (Homo) & Nonsense & Y197X & & N-terminal & $\mathrm{F}$ & $\mathrm{R}$ & Novel \\
\hline $15-17$ & C986A (Homo) & Nonsense & Y202X & & $\mathrm{N}$-terminal & $1 F, 2 M$ & $\mathrm{R}$ & Rees et al., 2001 \\
\hline \multirow[t]{2}{*}{18} & G1074A & Missense & S231N & \multirow{9}{*}{ * } & M1 & \multirow{2}{*}{$M$} & $\mathrm{R}$ & Novel \\
\hline & C1257A & Nonsense & S296X & & M3 & & $\mathrm{R}$ & Bellini et al., 2007 \\
\hline 19 & $\mathrm{C} 1128 \mathrm{~A}$ & Missense & Р250T & & $\mathrm{M} 1-\mathrm{M} 2$ & M & D & Saul et al., 1999 \\
\hline 20 & C1134T (Homo) & Missense & R252C & & $\mathrm{M} 1-\mathrm{M} 2$ & M & $\mathrm{R}$ & Novel \\
\hline 21,22 & G1141A (Homo) & Missense & G254D & & $\mathrm{M} 2$ & $2 \mathrm{M}$ & $\mathrm{R}$ & Novel \\
\hline 23 & C1174T & Missense & T265I & & M2 & M & $D$ & Novel \\
\hline $24-27$ & G1192A & Missense & R2710 & & $\mathrm{M} 2-\mathrm{M} 3$ & $2 \mathrm{~F}, 2 \mathrm{M}$ & $D$ & Shiang et al., 1993 \\
\hline 28,29 & G1404A & Missense & G342S & & M3-M4 & $1 \mathrm{~F}, 1 \mathrm{M}$ & D & Rees et al., 2001 \\
\hline 30 & G1555A (Homo) & Missense & $\mathrm{R} 392 \mathrm{H}$ & & M3-M4 & $M$ & $\mathrm{R}$ & Vergouwe et al., 1999 \\
\hline
\end{tabular}

Mat, Maternal; Pat, paternal; F, female; M, male; R, recessive; D, dominant. *Parental DNA not available; however, case 18's unaffected male sibling has only one of the heterozygous mutations, namely, S296X, indicating by default that S231N and S296X are present on different alleles.

startle response, which can provoke unprotected falls and result in injury, can remain throughout development and into adulthood (Andermann et al., 1980).

Previously identified dominant GLRA1 mutations are typically missense mutations predominantly located either in or near the M2 pore-lining domain of the GlyR $\alpha 1$ subunit (Saul et al., 1999; Harvey et al., 2008). The functional consequences of dominant GLRA1 mutations demonstrate that mutations in these regions exert dominant-negative effects by altering the allosteric signal transduction process coupling agonist binding to ionchannel gating (Harvey et al., 2008). By contrast, all nonsense and frameshift mutations in GLRA1 are associated with recessive cases of hyperekplexia with asymptomatic carrier parents, proving that hyperekplexia is not susceptible to haploinsufficiency (Brune et al., 1996; Rees et al., 2001). Functional studies of recessive missense mutations revealed a reduction in whole-cell currents, possibly because of the defects in GlyR trafficking or a decrease of membrane expression (Lynch et al., 1997; Rees et al., 2001; Humeny et al., 2002; Villmann et al., 2009).

Here, we present the systematic sequencing of GLRA1 in 88 unrelated hyperekplexia probands in an international screening program that identified 5 dominant and 14 recessive GLRA1 mutations within 30 index case families of which 12 are novel. A proportion of these novel mutations, including 10 recessive mutations, were characterized in a recombinant heteromeric GlyR $\alpha 1 \beta$ expression system. This study reveals that the pathophysiological mechanisms of dominant mutations differ from those of recessive mutations and that, contrary to established dogma, recessive hyperekplexia is decidedly more common than dominant hyperekplexia on a population basis. Last, we present a novel pathophysiological mechanism for hyperekplexia and neuronal ligand-gated channels: a dominant $\alpha 1$ subunit mutation (Y128C) that produces an open confirmation and tonic conductance through heteromeric $\alpha 1 \beta$ GlyRs.

\section{Materials and Methods}

Patients and cases. With informed consent procedures in place, patients with a clinical diagnosis of hyperekplexia were ascertained by referral from neurologists, pediatricians, or clinical geneticists from international centers. Eighty-eight unrelated index patients were included in this population study after phenotypic evaluation. Clinical inclusion criteria included a nonhabituating startle response (positive nose tap test), history of infantile hypertonicity, and an exclusion of phenocopies such as startle epilepsy (Harvey et al., 2008). The gender of patients is indicated in Table 1.

Molecular genetics. The exons and exon-intron boundaries of GLRA1 were amplified using primers designed using the Primer 3.0 program (MIT). To avoid allelic dropout, all primers were placed in intronic regions that were devoid of single-nucleotide polymorphisms (SNPs) as revealed by National Center for Biotechnology Information (NCBI) databases (Tester et al., 2006). Following conventional PCR protocols with patient DNA, PCR products were purified with QIAquick purification kit (QIAGEN) and directly sequenced using BigDye terminators and an ABI3100 automated Sequencer (Applied Biosystems). Frequency of SNPs or mutations was assessed in 100 control alleles using restriction fragment length polymorphism if a suitable restriction enzyme was available, or matrix-assisted laser desorption ionization time-of-flight mass spectrometry using protocols modified from those of Powell et al. (2004). In addition to sequence screening, all DNA samples were screened for large deletions or insertions in GLRA1 using a MLPA (Multiplex Ligation-Dependent Probe Amplification) DNA detection kit following the manufacturer's protocol (MRF). For samples with the recurrent $\Delta$ Exon1-7 deletion mutation, the previously identified breakpoints were confirmed by using a PCR assay as previously described (Becker et al., 2006).

Mutagenesis and expression of constructs. Full-length human GlyR $\alpha 1$ and $\beta$ subunits were cloned into the vector pRK5 (Rees et al., 2006) and the pIRES2-EGFP plasmid vector (Clontech), respectively. Mutations were introduced into pRK5-hGlyR $\alpha 1$ using the QuikChange SiteDirected Mutagenesis kit (Stratagene). All expression constructs were confirmed by sequencing the entire coding region. Wild-type (WT) or mutant GlyR $\alpha 1$ subunits were transiently expressed in human embryonic 
kidney (HEK293) cells using Effectene transfection reagent (QIAGEN) or a calcium phosphate technique. For experiments involving homomeric GlyRs, pRK5-hGlyR $\alpha 1$ was cotransfected with the pEGFPC1 vector (Clontech) as a transfection marker in a 1:1 ratio. For heteromeric GlyRs, $\alpha 1$ and $\beta$ subunit expression constructs were transfected at a DNA ratio of 1:10. Homomeric $\alpha 1$ subunit or heteromeric $\alpha 1 \beta$ subunit GlyRs were distinguished by applying $100 \mu \mathrm{M}$ picrotoxin (PTX), which inhibits homomeric GlyRs but not heteromeric GlyRs (Lynch, 2004). Twenty-four hours after transfection, cells were washed twice, and recordings were made at room temperature (RT) within the following 24-48 h.

Electrophysiology patch clamping. Whole-cell patch-clamp recordings were performed on HEK293 cells placed in an external solution consisting of the following (in mM): $140 \mathrm{NaCl}, 5 \mathrm{KCl}, 2 \mathrm{CaCl}_{2}, 1 \mathrm{MgCl}_{2}, 10$ HEPES, 10 glucose, adjusted to $\mathrm{pH} 7.4$ with $\mathrm{NaOH}$. Electrodes were pulled from borosilicate glass hematocrit tubing (Vitrex) with a horizontal puller (P97; Sutter Instrument) and heat polished. The electrodes have resistances of 1-3 M $\Omega$ when filled with an internal pipette solution consisting of the following (in mM): $145 \mathrm{CsCl}, 2 \mathrm{CaCl}_{2}, 2 \mathrm{MgCl}_{2}, 10$ HEPES, 10 EGTA, adjusted to $\mathrm{pH} 7.4$ with $\mathrm{NaOH}$. Glycine-gated currents were recorded using the whole-cell patch-clamp configuration at a holding potential of $-40 \mathrm{mV}$. Solutions were applied to whole cells via gravity through parallel microtubules. The perfusion system was under the control of a manual micromanipulator and solution exchange was routinely complete within $100 \mathrm{~ms}$ between adjacent tubes. Currents were digitized at $1 \mathrm{kHz}$, filtered at $500 \mathrm{~Hz}$, and digitally recorded on a computer using an Axopatch 1D amplifier and pClamp9 software (Molecular Devices). The half-maximal concentration $\left(\mathrm{EC}_{50}\right.$ ) and Hill coefficient $\left(n_{\mathrm{H}}\right)$ values were calculated for individual glycine concentration-response relationships using the Hill equation fitted by a nonlinear leastsquares analysis (SigmaPlot 9.0). For quantifying tonic channel activity, voltage ramps were applied from -80 to $+80 \mathrm{mV}$ over a $2 \mathrm{~s}$ interval, from a holding potential of $-40 \mathrm{mV}$.

High-content screening system. Cells were imaged using an automated high-content screening system using YFP-I152L fluorescence as an indicator of $\mathrm{Cl}^{-}$influx rate (Kruger et al., 2005; Gilbert et al., 2009). Briefly, HEK293 cells were cotransfected with mutant or WT pRK5-hGlyR $\alpha 1$ and pcDNA3.1-YFP-I152L and plated into a 384-well plate $\left(\sim 2.5 \times 10^{3}\right.$ cells/well). Within the following $24-32 \mathrm{~h}$, cells were incubated in $25 \mu \mathrm{l}$ of standard $\mathrm{NaCl}$ control solution ( $140 \mathrm{~mm} \mathrm{NaCl}, 5 \mathrm{~mm} \mathrm{KCl}, 2 \mathrm{~mm} \mathrm{CaCl}, 1$ $\mathrm{mm} \mathrm{MgCl}_{2}, 10 \mathrm{~mm}$ HEPES, and $10 \mathrm{~mm}$ glucose, $\mathrm{pH} 7.4$ using $\mathrm{NaOH}$ ) for $1 \mathrm{~h}$, and 10 different glycine concentrations were prepared in NaI solution (140 mM NaI, $5 \mathrm{~mm} \mathrm{KCl}, 2 \mathrm{~mm} \mathrm{CaCl}$, $1 \mathrm{~mm} \mathrm{MgCl}_{2}, 10 \mathrm{~mm}$ HEPES, and $10 \mathrm{~mm}$ glucose, $\mathrm{pH} 7.4$ using $\mathrm{NaOH}$ ). Fluorescence images of each well were obtained twice, before and after the application of $\mathrm{NaI}$ solution containing a varying concentration of glycine. The percentage fluorescent change (quench) was calculated using the following equation: ( $1-$ $\left.\left(F_{\text {final }} / F_{\text {init }}\right)^{*} 100\right)$, where $F_{\text {init }}$ is the initial (or control) fluorescence value before the application of glycine, and $F_{\text {final }}$ is the fluorescence level after the application of glycine. Concentration-response curves were constructed by pooling results from two wells exposed to the same glycine concentration. Averaged fluorescence values were used to construct a dose-response curve, which was fitted as described above (SigmaPlot 9.0). For each mutant, screening was replicated at least three times.

Biotinylation. Two days after transfection, surface expression of GlyR $\alpha 1$ subunits in HEK293 cells were investigated using a cell membraneimpermeable reagent Sulfo-NHS-LC-Biotin (Pierce Biotechnology) based on the manufacturer's protocol. Briefly, transfected HEK293 cells in a $100 \mathrm{ml}$ dish were washed twice with ice-cold PBS buffer and incubated in $10 \mathrm{ml}$ of PBS containing $0.25 \mathrm{mg} / \mathrm{ml}$ Sulfo-NHS-SS-biotin for 30 $\min$ at $4^{\circ} \mathrm{C}$ with gentle shaking. Excess biotinylating reagent was then removed by adding quenching solution (Pierce) followed by washing the cells twice with TBS (Pierce). Cells were lysed using a lysis buffer (Pierce) supplemented with protease inhibitors (Pierce). To remove the nonsoluble fraction, the lysate was centrifuged at $14,000 \times g$ for 15 min at $4^{\circ} \mathrm{C}$, and then the clear supernatant was removed for additional analysis. The total protein concentration was measured with a DC Protein Assay (Bio-Rad), and $50 \mu \mathrm{g}$ of total protein was removed for immunoblotting analysis. To separate biotin-labeled surface proteins, $500 \mu \mathrm{g}$ of protein was incubated with $125 \mu \mathrm{l}$ of immobilized biotin-binding protein, Neu-
trAvidin gels (Pierce) for $1 \mathrm{~h}$ at RT. The biotin protein attached beads were then washed four times with a wash buffer (Pierce). The surface proteins were isolated from NeutrAvidin linked biotin molecule by incubating with $100 \mu \mathrm{l}$ of a sample buffer containing $50 \mathrm{~mm}$ reducing reagent (DTT) for $1 \mathrm{~h}$ at RT. The surface proteins were then eluted by centrifugation at $1000 \times g$ for $2 \mathrm{~min}$. Total protein lysates were also obtained from cells transfected with mutant or WT GlyR $\alpha 1$ and $\beta$ subunits by lysing cells as described above, but without the biotinylation labeling. Proteins in the whole-cell lysates or cell surface proteins were analyzed by Western blotting with an antibody against the GlyR $\alpha 1$ subunit (1:1500; Millipore). An anti- $\beta$-actin antibody (1:7000; Abcam) was used as a control to confirm that intracellular proteins were not labeled with biotin. The intensity of the immunoreactivity signal was quantified with ImageJ software (http://rsb.info.nih.gov/ij).

Statistical analysis. Data were analyzed using SigmaPlot (version 9.0; Systat Software) or GraphPad Prism (version 3.02; GraphPad Software) and expressed as mean \pm SEM. Statistical significance was determined by Student's $t$ test and considered to be significant at $p<0.05$.

Molecular modeling. Structural modeling of GlyR $\alpha 1 \mathrm{WT}$ and mutant proteins was performed using a homology modeling pipeline built with the Biskit structural bioinformatics platform (Grünberg et al., 2007), which scans the entire Protein Data Bank (PDB) database for candidate homologies. The best match attained for GlyR $\alpha 1$ was based on $26 \%$ identity with the crystal structure of the Torpedo $\alpha_{1} \beta \gamma \alpha_{2} \varepsilon$ nicotinic ACh receptor (nAChR) (Unwin, 2005) (PDB: 2BG9). Our pipeline workflow incorporates the NCBI tools platform (Wheeler et al., 2007), including the BLAST program (Altschul et al., 1990) for similarity searching of sequence databases. T-COFFEE (Notredame et al., 2000) was used for alignment of the test sequence with the template. Homology models were generated over 10 iterations of the MODELLER program (Eswar et al., 2003). The Torpedo nAChR pentamer was subsequently used as the template for the assembly of the pentameric GlyR $(2 \alpha, 3 \beta)$, built by overlaying $\alpha 1$ (WT or mutant) and $\beta$ subunits on the subunit positions of the Torpedo nAChR pentamer using Chimera (Pettersen et al., 2004).

\section{Results}

\section{Mutation analysis of GLRA1 in hyperekplexia}

A total of 88 index cases with confirmed hyperekplexia were screened for genetic variation in the entire coding and splice site regions of GLRA1. All sequence variations were regarded as mutations after exclusion from a panel of human controls. Direct sequencing analysis revealed 19 mutations within 30 hyperekplexia index cases, of which 12 mutations were novel and 7 of the mutations were discovered in more than one index case (Table 1; supplemental Fig. $S 1 A-D$, available at www.jneurosci.org as supplemental material). The majority of index cases $(21$ of $30 ; 70 \%$ ) showed recessive inheritance, including four cases of compound heterozygote inheritance, thereby dramatically increasing the number of pathological recessive hyperekplexia alleles in the literature. Consistent with previous studies, all nonsense and deletion/frameshift mutations were associated with recessive cases of hyperekplexia, whereas missense mutations transmit in either a dominant or recessive manner depending on their relative position in the GlyR $\alpha 1$ subunit or coinheritance in compound heterozygotes (supplemental Fig. S1 D, available at www.jneurosci. org as supplemental material). Interestingly, recessive mutations were scattered throughout the $\alpha 1$ GlyR subunit, whereas dominant mutations were specifically clustered in and around the pore-lining M2 domain (supplemental Fig. S1 B, C, available at www.jneurosci.org as supplemental material).

\section{Electrophysiological analysis of GlyR $\alpha 1$ subunit mutants}

To determine the functional effects of GLRA1 mutations, WT or mutant human $\alpha 1$ subunits were transiently expressed in HEK 293 cells either as homomeric $\alpha 1$ or heteromeric $\alpha 1 \beta$ GlyRs. 
A

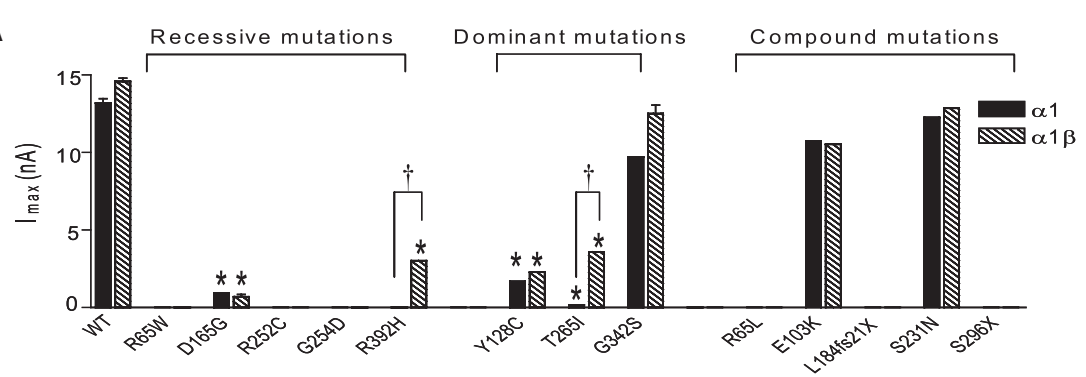

B

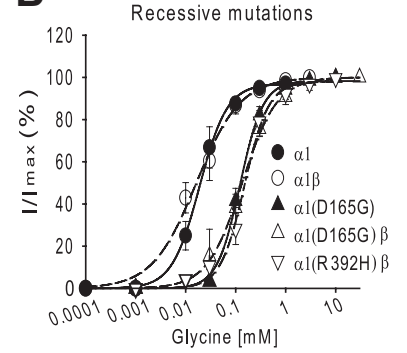

C

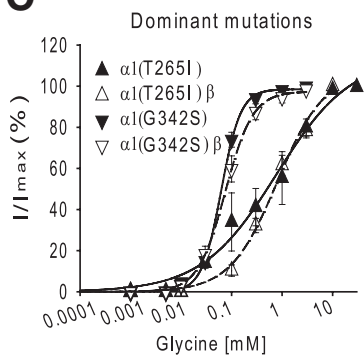

D

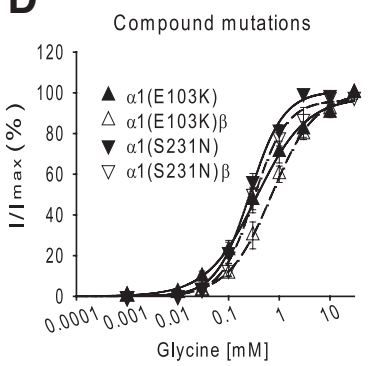

Figure 1. $I_{\max }$ values at saturating glycine responses and dose-response characteristics of glycine-induced currents. $\boldsymbol{A}$, Maximal GlyR currents were obtained from HEK293 cells expressing WT or mutant homomeric $\alpha 1$ subunit GlyRs or heteromeric $\alpha 1 \beta$ GlyRs at saturating glycine concentrations (up to $30 \mathrm{~mm}$ ). HEK293 cells that did not generate any glycine-induced currents were excluded from the analysis. ${ }^{*} p<0.001$ versus WT; ${ }^{\dagger} p<0.01$ versus heteromer. $\boldsymbol{B}-\boldsymbol{D}$, The currents are shown as a percentage of the maximal current for each cell. The solid-line dose-response curves represent GlyR $\alpha 1$ homomers, whereas the dashed curves represent GlyR $\alpha 1 \beta$ heteromers. Error bars indicate SEM.

\section{Recessive variants}

Most recessive mutations (R65W, R252C, G254D) failed to generate currents either as homomeric or heteromeric GlyRs on application of $30 \mathrm{~mm}$ glycine (Fig. $1 A$, Table 2). One novel recessive mutation, D165G, induced detectable, but significantly reduced, maximum currents compared with WT, regardless of whether it was expressed as a homomer or heteromer. Notably, the glycine sensitivity of this mutant was markedly decreased (Fig. $1 \mathrm{~B}$; supplemental Fig. S2, available at www.jneurosci.org as supplemental material). The mutation $\mathrm{R} 392 \mathrm{H}$, identified as a homozygous recessive mutation in this study, was previously identified as being part of a compound heterozygote (R252H and $\mathrm{R} 392 \mathrm{H}$ ) (Vergouwe et al., 1999). Consistent with previous findings, expression of homomeric R392H mutant in HEK293 cells did not induce detectable currents $(n=20$ for whole-cell patching clamp; $n>2000$ cells for automated screening). However, coexpression with GlyR $\beta$ produced small but detectable currents $\left(I_{\max }=3023 \pm 925 \mathrm{pA} ; n=6\right)($ Fig. $1 A)$ that exhibited a significant increase in glycine $\mathrm{EC}_{50}$.

\section{Dominant variants}

Two novel dominant mutations, Y128C and T265I, showed dramatically reduced $I_{\max }$ values compared with WT heteromers (Fig. 1 A, Table 2). When expressed as $\alpha 1^{\mathrm{T} 265 \mathrm{I}}$ homomers, $30 \mathrm{~mm}$ glycine consistently generated currents with magnitudes $\sim 1 \%$ of WT current levels $\left(I_{\max }=165.44 \pm 36.6 \mathrm{pA} ; n=11 ; p<0.0001\right.$ vs WT). In contrast, when $30 \mathrm{~mm}$ glycine was applied to untransfected cells, no significant deviation in baseline current was ever observed ( $n>20$ gigasealed cells). The $n_{\mathrm{H}}$ value for $\alpha 1^{\text {T265I }}$ was significantly reduced, suggesting a disruption in gating efficacy (Colquhoun, 1998). However, when $\alpha 1^{\text {T265I }}$ was coexpressed with the $\beta$ subunit, the heteromeric glycine-mediated current was easily detectable and a 20 -fold increase in $I_{\max }$ was observed, although it remained significantly less than values observed in WT heteromers (Fig. 1A). In both homomeric and heteromeric configurations, T265I exhibited a significantly increased glycine $\mathrm{EC}_{50}$ (Fig. $1 C$, Table 2). Last, the recurrent mutation G342S, identified in two dominant index cases in this study and in previous studies (Rees et al., 2001), induced $I_{\max }$ currents and a glycine $\mathrm{EC}_{50}$ value comparable with that of WT GlyRs.

\section{Compound heterozygosity}

Hemizygous missense mutations, R65L, E103K, and S231N, coinherited with large deletions or nonsense mutations (in cases 7, 10, and 18, Table 1; supplemental Fig. S1 $D$, available at www.jneurosci.org as supplemental material) were also analyzed. R65L, coinherited with $\Delta$ Exon4-7, generated no current when expressed in HEK293 cells (Fig. 1A). This was predictable, since the positively charged R65 residue is an important glycine binding determinant (Grudzinska et al., 2005). By contrast, mutations E103K and S231N behaved differently from conventional recessive missense mutants despite being coinherited with frameshift (L184fs21X) and nonsense (S296X) mutations, respectively (supplemental Fig. S1 D, available at www.jneurosci.org as supplemental material). This should mean that $\alpha 1^{\mathrm{E} 103 \mathrm{~K}}$ and $\alpha 1^{\mathrm{S} 231 \mathrm{~N}}$ are the only functional copies of GlyR subunits in vivo. Surprisingly, both S231N and E103K, either as homomeric $\alpha 1$ or heteromeric $\alpha 1 \beta$ GlyRs, generated fully functional channels with $I_{\max }$ currents compatible with WT GlyRs at $30 \mathrm{~mm}$ glycine (Fig. $1 A$ ). However, the $\mathrm{EC}_{50}$ for glycine was significantly increased in $\alpha 1^{\mathrm{E} 103 \mathrm{~K}} \beta$ and $\alpha 1^{\text {S231N }} \beta$ GlyRs relative to WT $\alpha 1 \beta$ controls (Fig. $1 D$, Table 2; supplemental Fig. S2, available at www.jneurosci.org as supplemental material). Unsurprisingly, both S296X and L184fs21X mutants failed to generate functional channels as either homomers or heteromers.

\section{GlyR $\alpha 1$ T265 is a novel determinant of PTX sensitivity}

PTX, a botanical alkaloid, potently inhibits homomeric $\alpha 1-3$ GlyRs, whereas the corresponding heteromeric $\alpha \beta$ GlyRs are insensitive (Hawthorne et al., 2006). For WT and all other $\alpha 1$ GlyR mutants investigated in this study, PTX $(100 \mu \mathrm{M})$ strongly inhibited currents in cells expressing homomeric $\alpha 1$ GlyRs, but not heteromeric $\alpha 1 \beta$ GlyRs. One exception to this was the heteromeric $\alpha 1{ }^{\text {T265I }} \beta$ GlyR mutant, which displayed an anomalously high PTX sensitivity (Fig. 2 A). Individual PTX dose-responses in the $\alpha 1^{\mathrm{T} 265 \mathrm{I}} \beta$ GlyR were fitted with an averaged half-maximal inhibitory concentration $\left(\mathrm{IC}_{50}\right)$ of $12.7 \pm 0.82 \mu \mathrm{M}$ and an $n_{\mathrm{H}}$ of $1.126 \pm 0.103$ (Fig. $2 A$ ). This $\mathrm{IC}_{50}$ is significantly lower than the corresponding values of the heteromeric $\alpha 1 \beta$ GlyR, which have previously been determined at $219 \pm 28 \mu \mathrm{M}$ (Hawthorne et al., 2006). Site-directed mutagenesis studies have demonstrated that M2 structure is an important constraint for the antagonistic activity of PTX as mutations to various GlyR $\alpha 1$ pore-lining residues, G254, T258, S267, R271, reduce the inhibitory potency of PTX (Shan et al., 2001; Dibas et al., 2002). Structural analysis predicted that T265 forms a part of pore-lining residues, along with G254, T258, and S267, and plays an important role for maintaining a minimum pore diameter by forming a hydrophobic 
Table 2. Functional analysis of novel hyperekplexia mutations

\begin{tabular}{|c|c|c|c|c|c|c|}
\hline & & Homomeric $\alpha 1$ & & Heteromeric $\alpha 1 \beta$ & & \\
\hline & $\alpha 1$ & Glycine $\mathrm{EC}_{50}(\mathrm{~mm})$ & $n_{\mathrm{H}}$ & Glycine $\mathrm{EC}_{50}(\mathrm{~mm})$ & $n_{\mathrm{H}}$ & Functional effects \\
\hline$A D$ & WT & $20.74 \pm 7.27(6)$ & $1.86 \pm 0.3$ & $23.24 \pm 7.59(6)$ & $1.17 \pm 0.31$ & \\
\hline & Y128C & - & - & - & - & Tonic opening of channel \\
\hline & $\mathrm{T} 265 \mathrm{I}$ & $302.37 \pm 97.38^{*}(3)$ & $0.84 \pm 0.15$ & $774.83 \pm 219.53^{*}(6)$ & $1.01 \pm 0.1$ & Shift of $\mathrm{EC}_{50}$ \\
\hline & G342S & $62.26 \pm 6.0(6)$ & $2.40 \pm 0.18$ & $84.07 \pm 11.69(11)$ & $1.8 \pm 0.24$ & Rare SNP \\
\hline Recessive & R65W & - & - & - & - & Trafficking \\
\hline & D165G & $145.33 \pm 23.20^{\ddagger}(6)$ & $1.49 \pm 0.14$ & $212.88 \pm 56.83^{\dagger}(4)$ & $1.66 \pm 0.4$ & Trafficking \\
\hline & $\mathrm{R} 252 \mathrm{C}$ & - & - & - & - & Trafficking \\
\hline & G254D & - & - & - & - & Trafficking \\
\hline & $\mathrm{R} 392 \mathrm{H}$ & - & - & $164.79 \pm 35.73^{\dagger}(6)$ & $2.07 \pm 0.21$ & Trafficking \\
\hline Compound & R65L\# & - & - & - & - & No current \\
\hline & $\Delta$ Exons 4-7 & ND & & & & \\
\hline Compound & E103K & $540.7 \pm 148.3^{*}(8)$ & $1.05 \pm 0.13$ & $757.52 \pm 147.72^{*}(6)$ & $1.11 \pm 0.05$ & Shift of $\mathrm{EC}_{50}$ \\
\hline & L184fs21X & - & - & - & - & Trafficking \\
\hline Compound & S231N & $262.15 \pm 31.89^{\dagger}(5)$ & $1.32 \pm 0.16$ & $383.81 \pm 132.57^{\dagger}(5)$ & $1.75 \pm 0.39$ & Shift of $\mathrm{EC}_{50}$ \\
\hline & S296X & - & - & - & - & Trafficking \\
\hline
\end{tabular}

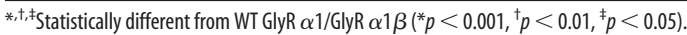

A

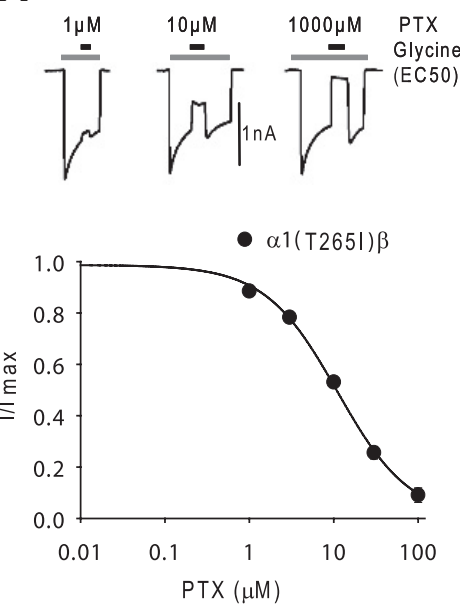

B

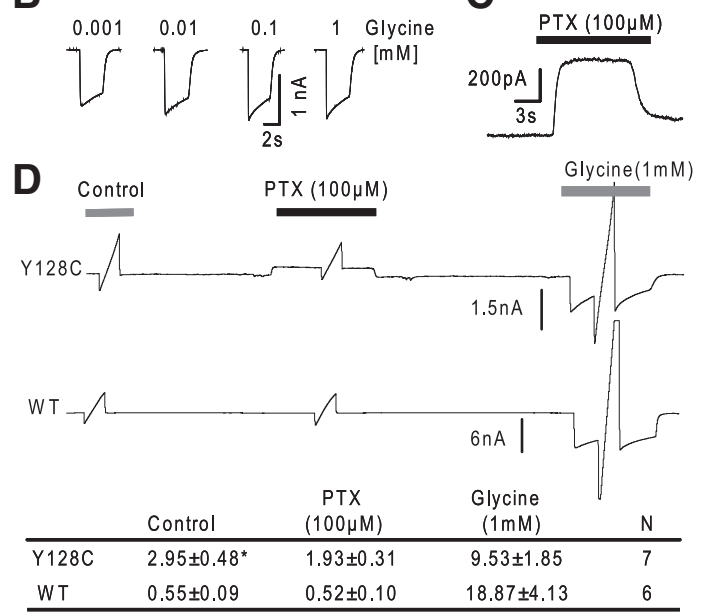

E

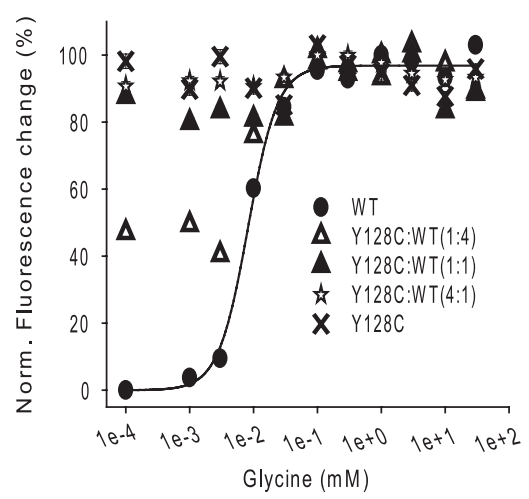

Figure 2. Novel effects of dominant mutations identified in this study. A, The GlyR $\alpha 1^{\text {T2651 }}$ mutation converted PTX-insensitive heteromeric $\alpha 1^{\text {T2651 }} \beta$ GlyRs to PTX-sensitive receptors. Top, Examples of PTX current inhibition produced by $\alpha 1^{\text {T265I }} \beta$ GlyRs when an increasing concentration of PTX was applied in the presence of an $\mathrm{EC}_{50}$ concentration of glycine. Bottom, Inhibitory dose-response curve. Parameters of best fit are given in the text. $\boldsymbol{B}-\boldsymbol{E}, \alpha 1^{\mathrm{Y} 128 \mathrm{C}}$ GlyRs generated tonic currents when expressed in HEK293 cells as either homomeric or heteromeric GlyRs. $\boldsymbol{B}$, Examples of glycine-induced currents produced by $\alpha 1^{Y 128 C}$. C, A representative trace of a decrease in inward current induced by PTX (100 $\mu \mathrm{m}$ ) in the absence of glycine. For WT, no detectable current was induced by PTX or in the absence of agonist. $\boldsymbol{D}$, Current-voltage relationship of WT/Y128C receptors. I-V curves were measured by whole-cell patch-clamp recordings (1) in the absence of agonist/antagonist (control), (2) in the presence of $100 \mu \mathrm{M}$ PTX, and (3) in the presence of $1 \mathrm{~mm}$ glycine. $\alpha 1{ }^{\mathrm{Y} 128 \mathrm{C}}$ shows unusually large resting conductances in the absence of glycine (control). ${ }^{*} p<0.001$ versus WT. $E$, Glycine responses of 2000 cells transfected with $\alpha 1^{\mathrm{Y} 128 \mathrm{C}}$ CDNA, or with combinations of $\alpha 1^{\mathrm{Y} 128 \mathrm{C}}$ CDNA with $\alpha 1^{\mathrm{WT}}$ in different ratios (Y128C:WT, 1:1, 2:1, or 4:1).

bond with Q266 in adjacent M2 domain (Miyazawa et al., 2003). T265 in the M2 domain represents a novel determinant of PTX sensitivity.

\section{GlyR $\alpha 1$ Y128C causes spontaneously open channels}

The novel variant $\mathrm{Y} 128 \mathrm{C}$ is the second dominant hyperekplexia mutation found in the LBD (supplemental Fig. S1C, available at www.jneurosci.org as supplemental material). The only previously reported LBD dominant mutation, R218Q, decreases both total and cell surface expression levels (Miraglia Del Giudice et al., 2003). By contrast, the Y128C mutant formed spontaneously opening channels when expressed as either homomeric $\alpha 1^{\mathrm{Y} 128 \mathrm{C}}$ or heteromeric $\alpha 1^{\mathrm{Y} 128 \mathrm{C}} \beta$ GlyRs (Fig. 2 B). Application of $100 \mu \mathrm{M}$ PTX, but not $10 \mu \mathrm{M}$ strychnine, significantly reduced inward currents ( $472 \pm 71 \mathrm{pA} ; n=25)$ in the absence of glycine, suggesting the closure of spontaneously open channels (Fig. 2C). Furthermore, the slope conductance between -80 and $+80 \mathrm{mV}$ for $\mathrm{Y} 128 \mathrm{C}$ in the absence of glycine was significantly higher com- pared with WT (Y128C, $2.95 \pm 0.48 \mathrm{nS}, n=7$; WT, $0.55 \pm 0.09$ $\mathrm{nS}, n=6$; $p<0.001)$ (Fig. $2 D)$. Application of PTX $(100 \mu \mathrm{M})$ reduced the leak conductance of Y128C by $35 \%$, whereas resting conductances for WT were not affected by PTX. As expected from the smaller glycine current produced by Y128C compared with WT (Fig. 1A), application of $1 \mathrm{~mm}$ glycine resulted in smaller slope conductance in Y128C than in WT. Since this is a dominant mutation, we sought to investigate how leak magnitude changed when $\alpha 1^{\text {Y128C }}$ coexpressed with WT subunits. Thus, $\alpha 1^{\text {Y128C }}$ was mixed with different ratios of WT $\alpha 1$ and coexpressed with the $\mathrm{I}^{-}$-sensitive $\mathrm{YFP}^{\mathrm{I1} 52 \mathrm{~L}}$. Using an automated live-cell imaging system, the glycine dose-response characteristics were determined by quantifying the fluorescence change after the application of increasing concentrations of glycine (0.001-10 $\mathrm{mm})$. After the application of a saturating glycine concentration ( $1 \mathrm{mM}),>90 \%$ of fluorescent cells transfected with $\alpha 1$ and $\mathrm{YFP}^{\mathrm{I} 152 \mathrm{~L}}$ displayed fluorescence quench, indicating $>90 \%$ of cotransfection efficiency of $\alpha 1$ and $\mathrm{YFP}^{\mathrm{I} 152 \mathrm{~L}}$. The $\mathrm{EC}_{50}$ of WT $\alpha 1$ 

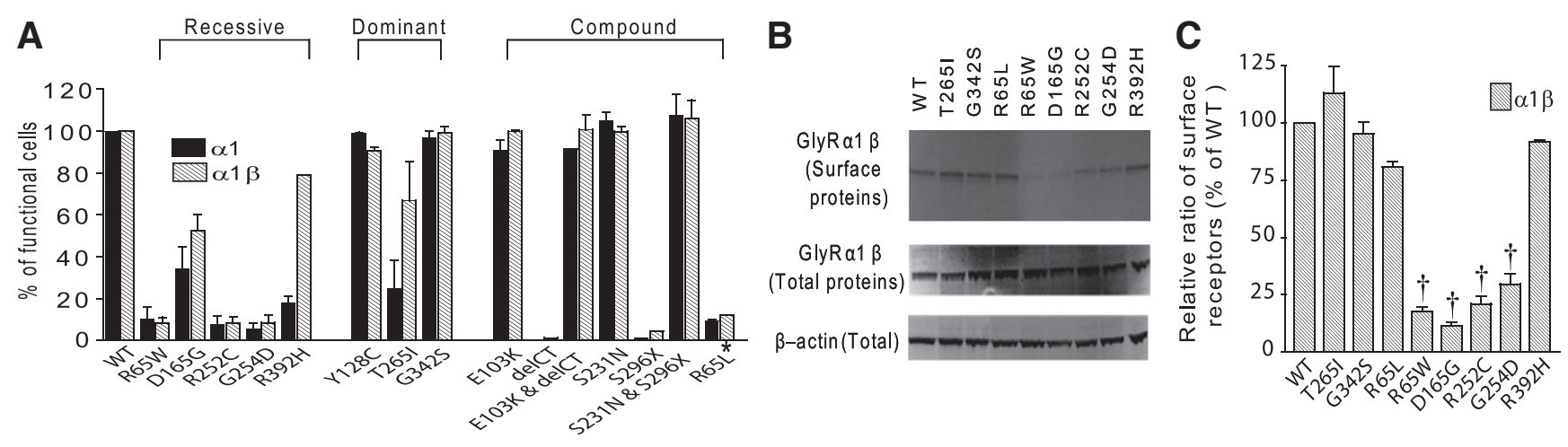

Figure 3. Expression level of mutant GlyRs. A, The WT human GlyR $\alpha 1$ subunit or hyperekplexia mutants were expressed in HEK293 cells, either as homomeric or heteromeric GlyRs, together with YFP. The number of cells expressing functional mutant channels was quantified (and compared with that of the WT subunit) using a YFP-based screening assay. Fluorescent cells that showed $>20 \%$ of fluorescent change (quench) after the application of a saturating concentration of glycine were considered to be expressing functional GlyRs. *R65L is coinherited with $\Delta$ Exon4-7 in case 7 (Table 1). $\boldsymbol{B}$, Biotinylation assay showed that recessive mutants were expressed in similar levels of whole-cell protein expression (total) but reduced cell surface protein expression (surface) compared with WT receptors. C, Surface expression of the GlyR $\alpha 1$ mutants was quantified using ImageJ software and expressed as a percentage of WT GlyR $\alpha 1$. ${ }^{\dagger} p<0.0001$. Fifty micrograms of protein lysates were loaded in the each lane. Error bars indicate SEM.

GlyRs was similar to that produced by patch clamp $(19.3 \pm 3.4$ $\mu \mathrm{M}$ for YFP imaging, $n>2000 ; 20.7 \pm 7.3 \mu \mathrm{M}$ for electrophysiology, $n=6)$. Cells transfected with Y128C $(n>2000)$ displayed a maximum fluorescent change in the absence of glycine (Fig. $2 E$ ). Cells transfected with an equal amount of Y128C and WT generated dose-response curves similar to that of cells expressing Y128C, confirming the dominant effect of Y128C on GlyR function.

\section{Defective subcellular localization is the major mechanism associated with recessive GLRA1 mutations}

For each mutant, the number of cells expressing functional GlyRs was determined using a live-cell imaging system in which HEK293 cells were cotransfected with mutant or WT GlyR $\alpha 1$ plus YFP. Screening of $>1500$ cells transfected with each different GlyR mutants demonstrated that recessive mutations display no change in florescence intensity indicating significant reduction in the number of functional channels, further supporting the absence of glycine-mediated currents in cells expressing these mutants (Fig. 3A). Dominant mutations Y128C and T265I and hemizygous missense mutations E103K and S231N displayed an expression level comparable with that of WT GlyRs. To investigate the effects of coexpression of compound heterozygote mutations, we screened $>2000$ HEK cells cotransfected with compound mutations (S296X plus S231R/E103K plus L184fs21X). Both S296X and L184fs21X mutants show loss of function; however, they did not demonstrate any dominant-negative effects on the expression of S231R or E103K, respectively (Fig. 3A). Previously, S296X was reported to exert dominant-negative effects on the WT GlyR $\alpha 1$ subunit by reducing $\mathrm{Cl}^{-}$current density from $\sim 170 \mathrm{pA} / \mathrm{pF}$ (WT) to $\sim 100 \mathrm{pA} / \mathrm{pF}$ (S296X plus WT) (Bellini et al., 2007). However, when HEK293 cells were transfected with S296X and WT GlyR $\alpha 1$ in the YFP automated system $(n>2000)$, no significant change in $\mathrm{EC}_{50}$ or maximum current was observed compared with WT alone.

\section{Biotinylation labeling assays}

Hyperekplexia mutants with low-level expression of functional GlyRs were further investigated for the surface expression. Biotinylation of cell surface proteins revealed that the reduction in the number of functional channels observed in recessive mutants is attributable to the decreased cell surface expression of GlyRs (Fig. $3 B, C)$. Although the whole-cell expression of recessive mutants was comparable with that of WT GlyR $\alpha 1$, cell surface expression levels were significantly decreased. These data provide additional support for a previous study on the mechanisms of recessive mutations (Villmann et al., 2009). As expected, mutants T265I and $\mathrm{R} 392 \mathrm{H}$ displayed robust cell surface expression. The hemizygous mutation, R65L, identified in a compound heterozygous case (Table 1, case 7), generated no current but had similar levels of surface protein expression compared with WT. By contrast, the recessive mutation $\mathrm{R} 65 \mathrm{~W}$ showed a significant reduction in the level of surface protein expression but a similar level of wholecell expression. The possible explanation for this residue-specific cell surface difference is described below in the molecular modeling section. For the dominant T265I mutation, there was no alteration in the cell surface expression indicating that the decreased level of T265I GlyRs is attributable to nonfunctional surface-targeted channels rather than a reduction in surface expression.

\section{Molecular modeling provides converging evidence with functional assays}

Structural modeling findings for GlyR $\alpha 1$ mutants are summarized in supplemental Table S1 (available at www.jneurosci.org as supplemental material) and Figure $4 A$. Several mutations are predicted to result in the marked rearrangement of TM regions, including Y128C and T265I, which both produce low currents (Fig. 4 B). Conversely, E103K and S231N mutants are predicted to result in only minor rearrangements of TM regions, consistent with these mutants showing robust glycine-gated currents (supplemental Fig. S3A, available at www.jneurosci.org as supplemental material). Relaxation of the $\beta$-strand structure in the vicinity of the glycine binding site was predicted for E103K, S231N, T265I, and R392H, and this could explain the observed increases in $\mathrm{EC}_{50}$ in our functional assays. By contrast, the R65W, R252C, and G254D mutants displayed extension of either the M2 or M3 helix into the LBD interface, possibly explaining their lack of cell membrane expression. The main predicted differences between R65L and R65W models can be observed in the $\beta$-strand structure of the extracellular domain, which is enhanced in R65W compared with R65L and WT (supplemental Fig. S3B, available at www.jneurosci.org as supplemental material). Comparisons of the models of heteropentameric GlyRs $\alpha 1 \beta$ and GlyR $\alpha 1^{\mathrm{Y} 128 \mathrm{C}} \beta$ (Fig. $4 C$ ) revealed that Y128C is predicted to disrupt the packing of the $\alpha 1$ subunit M2 and M3 helices and, as a result, 
cause disorder of pore architecture. Y128C produced a marked rearrangement of the M2 helix, resulting in a general slackening of the pore and loss of intimate contact between the key porelining residues, R252, G254, and T265 (Fig. $4 D$ ). Thus, it appears that the introduction of a short $\alpha$-helix in the LBD (supplemental Fig. S3C, available at www. jneurosci.org as supplemental material) at residues $142-145$ by the Y128C mutation disrupts $\alpha 1 \beta$ subunit interactions. This region is predicted to operate in conjunction with residues $183-185$, on the other side of the $\alpha 1$ subunit at the interface between the LBD and TM domains, in coordination of $\alpha \beta$ subunit packing.

\section{Discussion}

This study represents by far the largest multicenter screening program in hyperekplexia to date with concomitant functional characterization of GLRA1 mutations in hyperekplexia. Our direct sequencing screening program of 88 index cases, collected over 15 years, has identified 19 GLRA1 mutations within 30 index cases, of which 21 cases were inherited in recessive or compound heterozygote modes (Table 1). This study increases the compendium of hyperekplexia associated GLRA1 mutations by $42 \%$ and effectively doubles the number of gene-positive index cases known in the literature. Consistent with previous studies, all nonsense and intragenic deletion mutations were associated with recessive cases of hyperekplexia, confirming that haploinsufficiency is not a feature of this disorder. Hyperekplexia has traditionally been considered to be a dominant disorder (Harvey et al., 2008) mostly driven by the identification of multiply affected dominant families with a linked bias of M2 mutations and the relative high frequency of $\mathrm{R} 271 \mathrm{Q} / \mathrm{L}$ alleles in Caucasians. However, several recent studies have provided increasing evidence for the contribution of recessive alleles, led by the high frequency of $\Delta$ Exon 1-7 as a founder effect in the Turkish and Kurdish populations (Gilbert et al., 2004; Becker et al., 2006; Sirén et al., 2006). This latest study confirms that, on an index case population basis, the recessive inheritance of hyperekplexia is more common than dominant, although they are closely matched in their relative contribution. Combined with the data from the second major gene mutated in hyperekplexia, the glycine transporter 2 gene, SLC6A5 (Rees et al., 2006), in which compound heterozygosity and homozygous mutant alleles predominate, then the balance moves toward hyperekplexia being a predominantly recessive disorder.

As revealed by previous studies (Harvey et al., 2008), the clinical phenotypes of patients with recessive mutations were not as severe as the phenotype of hyperekplexia in animal models, in which recessive mutations are associated with more severe out-

D

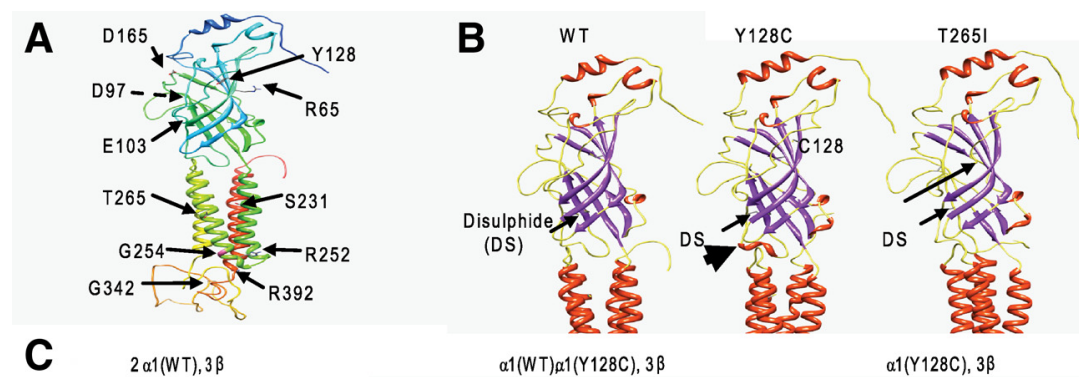

Structural modeling of GlyR $\alpha 1$ mutants. $\boldsymbol{A}$, GLRA1 mutations are shown on GlyR $\alpha 1$ subunit model. $\boldsymbol{B}$, Structura associated with low current; and relaxation of structure in the vicinity of the glycine binding site (regular long arrow), concomitant with observed increases in $\mathrm{EC}_{50}$. C, Pentameric form $(2 \alpha, 3 \beta)$ of $\alpha 1$ WT and $\mathrm{Y} 128 \mathrm{C}$ mutant, side view, extracellular sections 142-145 and 183-185 in red ( $\alpha$ conformation) and yellow ( $\beta$ conformation) as appropriate. R252 (where visible) at the cytoplasmic end, colored by element (blue), G254 (cytoplasmic end) in orange, and T265 (mid-membrane) in purple. D, Pentameric form, view from extracellular end, showing considerable disruption of subunit packing in the $\alpha 1 \mathrm{Y} 128$ C polypeptide, resulting in slackening around the ion pore and loss of intimate WT arrangement of key pore-lining residues G254 (orange), T265 (by element, red), and R252 (by element, blue).

comes leading to premature death (Buckwalter et al., 1994), indicating the likely existence of compensatory mechanisms in humans. Patients with recessive or null mutations in GLRA1, however, were associated with a spectrum of developmental disorders from motor delay to learning difficulties and challenging behavior (supplemental Table S2, available at www.jneurosci.org as supplemental material). Parents who were heterozygous carriers of the recessive/compound mutations did not present with clinical symptoms reminiscent of hyperekplexia. However, dominant mutations in index cases were associated with one affected parent within the nuclear family structure. In addition to novel insights of the molecular genetics of hyperekplexia, we have used high-throughput functional assays to reveal new pathogenic mechanisms associated with GLRA1 mutations. Of 12 novel GLRA1 mutations identified, all were submitted to functional analysis with the exception of the homozygous stop codon alleles 
(Y197X and Y202X) and large deletions (e.g., $\Delta$ Exon 4-7) in which the outcomes are unambiguous. This evidence-based approach is essential for establishing the pathophysiological principles underlying the abnormal startle response.

Our data essentially confirm the existence of three basic GlyR channelopathy mechanisms. (1) Cell surface expressed receptors that do not function correctly because of dominant mutations that compromise glycine ligand binding, alter apparent agonist sensitivity or cause chloride conductance defects; (2) recessive trafficking mutants in which pentameric assembly or transition through the transcription/translation processes conspire to cause a deficiency of cell surface targeting or insertion of GlyRs; (3) recessive null genotypes in which the creation of functional $\alpha 1 \beta$ pentamers is precluded, leading to a deficit in glycinergic neurotransmission in which compensatory mechanisms prevent a lethal clinical outcome. In addition, we have also determined that T265 in the M2 domain is an important determinant of the PTX activity, and that different substitutions at one position in GlyR $\alpha 1$ (R65L, R65W) result in quite different outcomes in functional analysis and molecular modeling. The only exception to the pathogenic trends was missense substitution G342S in which no indication of functional deficits was found, suggesting that this variation could simply be a rare SNP. Alternatively, given the location of this residue in the M3-M4 domain, perhaps G342S disrupts an as-yet-uncharacterized GlyR-accessory protein interaction.

Our study has also identified a new pathogenic mechanism in hyperekplexia: spontaneously opening GlyRs. A single dominant missense mutation, Y128C, in the GlyR $\alpha 1$ subunit LBD, resulted in tonic channel opening in the absence of agonist. Spontaneous channel activity has been recognized as a pathogenic mechanism in other episodic disorders including congenital muscle disease, cardiac arrhythmias, and hypokalemic periodic paralysis (Paavola et al., 2007; Sokolov et al., 2007; Treves et al., 2008). Previous GlyR $\alpha 1$ site-directed mutagenesis studies have also identified three residues causing spontaneously opening: D97R and F99A in the LBD and A288W in M3 (Mihic et al., 1997; Beckstead et al., 2002; Miller et al., 2008). Indeed, our structural modeling indicates that Y128 is located in close proximity to D97 and F99. Y128C, F99A, and D97R are all predicted to induce the same short $\alpha$-helix in the LBD directly overlying the TM regions suggesting a common mechanism of tonic channel opening. By contrast, A288W, which is located near the M3 extracellular boundary, appears to achieve tonic opening by an alternative mechanism. The large tryptophan residue projects outward from M3 and probably interferes with subunit packing. Although the precise mechanisms underlying the spontaneous channel activation are unclear at present, a recent study revealed that agonist binding to GlyR $\alpha 1$ initiates rearrangements of the LBD inner $\beta$-sheet to trigger additional movements for removing channel gating (Pless and Lynch, 2009). Since Y128 lies in loop E in the inner $\beta$-sheet, the tyrosine-to-cysteine substitution at residue 128 is expected to interrupt the $\beta$-sheet structure, thereby favoring the open conformation. How does spontaneous GlyR activity produce hyperekplexia? It might be expected that overactive GlyRs would chronically inhibit postsynaptic motor neurons. However, this would lead to a long-term dampening of motor neuron activity, which is not observed in hyperekplexia patients. Given that the carrier of Y128C has classical hyperekplexia symptoms, it is more likely that compensatory mechanisms restore normal ionic gradients and neuronal excitability and that the hyperekplexia phenotype is attributable to a reduced magnitude of glycine-activated inhibitory currents through Y128C mutant GlyRs.
This study has revealed novel and recurrent mutations combining this with high-throughput functional analysis to confirm pathophysiological and clinical conclusions. For GLRA1 genenegative patients, additional analysis awaits for SLC6A5, GLRB, $G P H N$, and other loci that have previously underlined the genetic heterogeneity and systems biology approach in hyperekplexia (Harvey et al., 2008). As molecular genetics enters the next technical revolution to third-generation sequencing and array-based analysis, it must not be forgotten that careful functional characterization of genotypes must accompany the sequencing data if the correct in vivo mechanisms are to be identified in hyperekplexia and other neurological disorders. Our functional analysis of the novel mutations revealed new mechanisms of action including the first description of GlyR tonic opening (Y128C), an important determinant of PTX binding (T265I), and the first mutations in the glycine binding site (R65) revealing substitution-specific effects on cell surface trafficking. Several lines of convergent evidence, including biophysical characterization, cell surface expression, and molecular modeling, all provide a pathophysiological basis for the dysfunction of an evolutionarily conserved, ancient startle response.

\section{References}

Altschul SF, Gish W, Miller W, Myers EW, Lipman DJ (1990) Basic local alignment search tool. J Mol Biol 215:403-410.

Andermann F, Keene DL, Andermann E, Quesney LF (1980) Startle disease or hyperekplexia: further delineation of the syndrome. Brain 103: 985-997.

Becker K, Hohoff C, Schmitt B, Christen HJ, Neubauer BA, Sandrieser T, Becker CM (2006) Identification of the microdeletion breakpoint in a GLRA1null allele of Turkish hyperekplexia patients. Hum Mutat 27:1061-1062.

Beckstead MJ, Phelan R, Trudell JR, Bianchini MJ, Mihic SJ (2002) Anesthetic and ethanol effects on spontaneously opening glycine receptor channels. J Neurochem 82:1343-1351.

Bellini G, Miceli F, Mangano S, Miraglia del Giudice E, Coppola G, Barbagallo A, Taglialatela M, Pascotto A (2007) Hyperekplexia caused by dominantnegative suppression of glyral function. Neurology 68:1947-1949.

Brune W, Weber RG, Saul B, von Knebel Doeberitz M, Grond-Ginsbach C, Kellerman K, Meinck HM, Becker CM (1996) A GLRA1 null mutation in recessive hyperekplexia challenges the functional role of glycine receptors. Am J Hum Genet 58:989-997.

Buckwalter MS, Cook SA, Davisson MT, White WF, Camper SA (1994) A frameshift mutation in the mouse alpha 1 glycine receptor gene (Glra1) results in progressive neurological symptoms and juvenile death. Hum Mol Genet 3:2025-2030.

Colquhoun D (1998) Binding, gating, affinity and efficacy: the interpretation of structure-activity relationships for agonists and of the effects of mutating receptors. Br J Pharmacol 125:924-947.

Dibas MI, Gonzales EB, Das P, Bell-Horner CL, Dillon GH (2002) Identification of a novel residue within the second transmembrane domain that confers use-facilitated block by picrotoxin in glycine alpha 1 receptors. J Biol Chem 277:9112-9117.

Eswar N, John B, Mirkovic N, Fiser A, Ilyin VA, Pieper U, Stuart AC, MartiRenom MA, Madhusudhan MS, Yerkovich B, Sali A (2003) Tools for comparative protein structure modeling and analysis. Nucleic Acids Res 31:3375-3380.

Gilbert DF, Wilson JC, Nink V, Lynch JW, Osborne GW (2009) Multiplexed labeling of viable cells for high-throughput analysis of glycine receptor function using flow cytometry. Cytometry A 75:440-449.

Gilbert SL, Ozdag F, Ulas UH, Dobyns WB, Lahn BT (2004) Hereditary hyperekplexia caused by novel mutations of GLRA1 in Turkish families. Mol Diagn 8:151-155.

Grudzinska J, Schemm R, Haeger S, Nicke A, Schmalzing G, Betz H, Laube B (2005) The beta subunit determines the ligand binding properties of synaptic glycine receptors. Neuron 45:727-739.

Grünberg R, Nilges M, Leckner J (2007) Biskit—a software platform for structural bioinformatics. Bioinformatics 23:769-770. 
Harvey RJ, Topf M, Harvey K, Rees MI (2008) The genetics of hyperekplexia: more than startle! Trends Genet 24:439-447.

Hawthorne R, Cromer BA, Ng HL, Parker MW, Lynch JW (2006) Molecular determinants of ginkgolide binding in the glycine receptor pore. J Neurochem 98:395-407.

Humeny A, Bonk T, Becker K, Jafari-Boroujerdi M, Stephani U, Reuter K, Becker CM (2002) A novel recessive hyperekplexia allele GLRA1 (S231R): genotyping by MALDI-TOF mass spectrometry and functional characterisation as a determinant of cellular glycine receptor trafficking. Eur J Hum Genet 10:188-196.

Kruger W, Gilbert D, Hawthorne R, Hryciw DH, Frings S, Poronnik P, Lynch JW (2005) A yellow fluorescent protein-based assay for highthroughput screening of glycine and GABAA receptor chloride channels. Neurosci Lett 380:340-345.

Lynch JW (2004) Molecular structure and function of the glycine receptor chloride channel. Physiol Rev 84:1051-1095.

Lynch JW, Rajendra S, Pierce KD, Handford CA, Barry PH, Schofield PR (1997) Identification of intracellular and extracellular domains mediating signal transduction in the inhibitory glycine receptor chloride channel. EMBO J 16:110-120.

Mihic SJ, Ye Q, Wick MJ, Koltchine VV, Krasowski MD, Finn SE, Mascia MP, Valenzuela CF, Hanson KK, Greenblatt EP, Harris RA, Harrison NL (1997) Sites of alcohol and volatile anaesthetic action on $\mathrm{GABA}_{\mathrm{A}}$ and glycine receptors. Nature 389:385-389.

Miller PS, Topf M, Smart TG (2008) Mapping a molecular link between allosteric inhibition and activation of the glycine receptor. Nat Struct Mol Biol 15:1084-1093.

Miraglia Del Giudice E, Coppola G, Bellini G, Ledaal P, Hertz JM, Pascotto A (2003) A novel mutation (R218Q) at the boundary between the $\mathrm{N}$-terminal and the first transmembrane domain of the glycine receptor in a case of sporadic hyperekplexia. J Med Genet 40:e71.

Miyazawa A, Fujiyoshi Y, Unwin N (2003) Structure and gating mechanism of the acetylcholine receptor pore. Nature 423:949-955.

Notredame C, Higgins DG, Heringa J (2000) T-Coffee: a novel method for fast and accurate multiple sequence alignment. J Mol Biol 302:205-217.

Paavola J, Viitasalo M, Laitinen-Forsblom PJ, Pasternack M, Swan H, Tikkanen I, Toivonen L, Kontula K, Laine M (2007) Mutant ryanodine receptors in catecholaminergic polymorphic ventricular tachycardia generate delayed afterdepolarizations due to increased propensity to $\mathrm{Ca}^{2+}$ waves. Eur Heart J 28:1135-1142.

Pettersen EF, Goddard TD, Huang CC, Couch GS, Greenblatt DM, Meng EC, Ferrin TE (2004) UCSF Chimera-a visualization system for exploratory research and analysis. J Comput Chem 25:1605-1612.

Pless SA, Lynch JW (2009) Ligand-specific conformational changes in the alphal glycine receptor ligand-binding domain. J Biol Chem 284: 15847-15856.

Powell N, Dudley E, Morishita M, Bogdanova T, Tronko M, Thomas G (2004) Single nucleotide polymorphism analysis in the human phosphatase PTPrj gene using matrix-assisted laser desorption/ionisation time-offlight mass spectrometry. Rapid Commun Mass Spectrom 18:2249-2254.
Rees MI, Lewis TM, Vafa B, Ferrie C, Corry P, Muntoni F, Jungbluth H, Stephenson JB, Kerr M, Snell RG, Schofield PR, Owen MJ (2001) Compound heterozygosity and nonsense mutations in the alpha(1)-subunit of the inhibitory glycine receptor in hyperekplexia. Hum Genet 109: 267-270.

Rees MI, Lewis TM, Kwok JB, Mortier GR, Govaert P, Snell RG, Schofield PR, Owen MJ (2002) Hyperekplexia associated with compound heterozygote mutations in the beta-subunit of the human inhibitory glycine receptor (GLRB). Hum Mol Genet 11:853-860.

Rees MI, Harvey K, Pearce BR, Chung SK, Duguid IC, Thomas P, Beatty S, Graham GE, Armstrong L, Shiang R, Abbott KJ, Zuberi SM, Stephenson JB, Owen MJ, Tijssen MA, van den Maagdenberg AM, Smart TG, Supplisson S, Harvey RJ (2006) Mutations in the gene encoding GlyT2 (SLC6A5) define a presynaptic component of human startle disease. Nat Genet 38:801-806.

Saul B, Kuner T, Sobetzko D, Brune W, Hanefeld F, Meinck HM, Becker CM (1999) Novel GLRA1 missense mutation (P250T) in dominant hyperekplexia defines an intracellular determinant of glycine receptor channel gating. J Neurosci 19:869-877.

Shan Q, Haddrill JL, Lynch JW (2001) A single beta subunit M2 domain residue controls the picrotoxin sensitivity of alphabeta heteromeric glycine receptor chloride channels. J Neurochem 76:1109-1120.

Shiang R, Ryan SG, Zhu YZ, Hahn AF, O'Connell P, Wasmuth JJ (1993) Mutations in the alpha 1 subunit of the inhibitory glycine receptor cause the dominant neurologic disorder, hyperekplexia. Nat Genet 5:351-358.

Sirén A, Legros B, Chahine L, Misson JP, Pandolfo M (2006) Hyperekplexia in Kurdish families: a possible GLRA1 founder mutation. Neurology 67:137-139.

Sokolov S, Scheuer T, Catterall WA (2007) Gating pore current in an inherited ion channelopathy. Nature 446:76-78.

Tester DJ, Cronk LB, Carr JL, Schulz V, Salisbury BA, Judson RS, Ackerman MJ (2006) Allelic dropout in long QT syndrome genetic testing: a possible mechanism underlying false-negative results. Heart Rhythm 3:815-821.

Treves S, Jungbluth H, Muntoni F, Zorzato F (2008) Congenital muscle disorders with cores: the ryanodine receptor calcium channel paradigm. Curr Opin Pharmacol 8:319-326.

Unwin N (2005) Refined structure of the nicotinic acetylcholine receptor at 4A resolution. J Mol Biol 346:967-989.

Vergouwe MN, Tijssen MA, Peters AC, Wielaard R, Frants RR (1999) Hyperekplexia phenotype due to compound heterozygosity for GLRA1 gene mutations. Ann Neurol 46:634-638.

Villmann C, Oertel J, Melzer N, Becker CM (2009) Recessive hyperekplexia mutations of the glycine receptor alphal subunit affect cell surface integration and stability. J Neurochem 111:837-847.

Wheeler DL, Barrett T, Benson DA, Bryant SH, Canese K, Chetvernin V, Church DM, DiCuccio M, Edgar R, Federhen S, Geer LY, Kapustin Y, Khovayko O, Landsman D, Lipman DJ, Madden TL, Maglott DR, Ostell J, Miller V, Pruitt KD, et al. (2007) Database resources of the National Center for Biotechnology Information. Nucleic Acids Res 35:D5-D12. 